\title{
Cytoprotective effect of aquaporin 1 against lipopolysaccharide-induced apoptosis and inflammation of renal epithelial HK-2 cells
}

\author{
YIDUO WANG ${ }^{1}$, WENZHENG ZHANG ${ }^{1}$, GUANGZHE YU ${ }^{2}$, QIAN LIU ${ }^{1}$ and YINGYU JIN ${ }^{1}$ \\ Departments of ${ }^{1}$ Laboratory Diagnosis and ${ }^{2}$ Emergency Surgery, First Affiliated Hospital of Harbin Medical University, \\ Harbin, Heilongjiang 150001, P.R. China
}

Received October 9, 2017; Accepted February 2, 2018

DOI: $10.3892 /$ etm.2018.5992

\begin{abstract}
Sepsis is the most common underlying disease of disseminated intravascular coagulation. Acute kidney injury is a common and serious complications of sepsis. In the present study, a lipopolysaccharide (LPS)-induced human proximal tubule cell line (HK-2 cells) was selected as an in vitro model of septic acute kidney injury. The aim of the present study was to investigate whether aquaporin 1 (AQP-1) has a cytoprotective role in LPS-induced HK-2 cells. HK-2 cells were treated with $0-16 \mu \mathrm{g} / \mathrm{ml}$ LPS for $0-24 \mathrm{~h}$ to establish the in vitro model of sepsis. The results demonstrated that AQP-1 levels were the lowest of the eight AQP genes expressed in LPS-induced HK-2 cells. Prior to LPS treatment, HK-2 cells were transfected with pcDNA-AQP-1 or small interfering-AQP-1 and cell counting kint- 8 and flow cytometry assays were performed to assess cell viability and apoptosis rate, respectively. Changes in the expression of proinflammatory cytokines and chemokines, as well as important factors in the p38, extracellular signal-regulated kinase (ERK)1/2 and c-Jun N-terminal kinase (JNK) pathways, were assessed using reverse transcription-quantitative polymerase chain reaction, western blotting and ELISA, respectively. LPS treatment reduced viability, increased apoptosis and upregulated the expression of proinflammatory cytokines and chemokines in HK-2 cells. AQP-1 overexpression significantly reversed the effects of LPS and downregulated the expression of tumor necrosis factor- $\alpha$, interleukin (IL) -8 , IL-1 $\beta$ and monocyte chemoattractant protein-1. The p38, ERK1/2 and JNK pathways were activated
\end{abstract}

Correspondence to: Dr Yingyu Jin, Department of Laboratory Diagnosis, First Affiliated Hospital of Harbin Medical University, 23 Youzheng Street, Harbin, Heilongjiang 150001, P.R. China

E-mail: jinyingyuharbin@yahoo.com

Abbreviations: DIC, disseminated intravascular coagulation; LPS, lipopolysaccharide; AQP-1, aquaporin-1

Key words: disseminated intravascular coagulation, lipopolysaccharide, aquaporin 1, proinflammatory, apoptosis by LPS; however, the p38 and ERK1/2 pathways were blocked in AQP-1-overexpressing cells. AQP-1 overexpression was demonstrated to confer a survival advantage to LPS-injured HK-2 cells by controlling cell viability, apoptosis and inflammation, possibly via modulation of the p38 and ERK1/2 pathways. The results of the present study suggest that AQP-1 may be an effective treatment for acute kidney injury caused by sepsis.

\section{Introduction}

Disseminated intravascular coagulation (DIC) is a severe clinical condition that is caused by an underlying disease (1). The primary symptom is marked, continuous, widespread coagulation activation in circulating blood and the formation of microvascular thrombi (1). It has been reported that the underlying diseases that most commonly cause DIC include sepsis, aortic aneurysm, solid cancer, clotting disorders, acute promyelocytic leukemia and fibrinolysis (2). Sepsis-induced DIC models have been investigated by many studies (3-5).

Sepsis is characterized by physiologic, pathologic and biochemical abnormalities and has been identified as a life-threatening organ dysfunction (6). Sepsis arises when the body's response to infection injures its own tissues and organs and severe sepsis may cause serious complications (7), including acute kidney injury (8). Renal inflammation is one of the main pathological changes observed in acute kidney injury (9). Lipopolysaccharide (LPS), an important inflammatory factor, is the product of Gram-negative bacteria and has been used to establish renal inflammation models for the investigation of inflammation-associated renal diseases (10-12).

At present, 13 mammalian aquaporins (AQPs) have been identified, comprising two subgroups: Water-selective channels called orthodox AQPs and aquaglyceroporins that may be permeated by water, glycerol and other small molecules (13). Of the known AQPs, eight (AQP-1, -2, -3, -4, -6, -7, -8 and -11 ) are expressed in mammalian kidneys (13). However, the expression and functions of AQPs in LPS-induced HK-2 cells remain to be elucidated. In the present study, it was demonstrated that, of the eight AQPs expressed, AQP-1 had the lowest expression in LPS-induced HK-2 cells. It has been reported that AQP-1 overexpression in hepatocytes improves 
LPS-induced cholestasis (14). Furthermore, a previous study demonstrated that AQP-1 overexpression inhibits the aristolochic acid I-induced epithelial-mesenchymal transition of HK-2 cells, suggesting that AQP-1 may be a target for aristolochic acid nephropathy clinical therapy (15). AQP-1 overexpression significantly reversed LPS-induced damage in HK-2 cells and reduced the levels of tumor necrosis factor (TNF)- $\alpha$, interleukin (IL)-8, IL-1 $\beta$ and monocyte chemoattractant protein (MCP)-1. The results of the present study also revealed that LPS-induced p38 and extracellular signal-regulated kinase (ERK)1/2 pathways were blocked in AQP-1-overexpressing cells and were aggravated in AQP-1-knockdown cells. However, AQP-1 did not affect the c-Jun N-terminal kinase (JNK) pathway. These results suggest that AQP-1 is able to reverse the LPS-induced decrease in cell viability, increase in apoptosis and inflammation in HK-2 cells, possibly via the p38 and ERK1/2 pathways.

\section{Materials and methods}

Cell culture and transfection. Renal tubular epithelial HK-2 cells (a proximal tubular cell line derived from normal kidney; CRL-2190), were purchased from the ATCC (Manassas, VA, USA) and incubated at $37^{\circ} \mathrm{C}$ for 2 days in a humidified atmosphere containing $5 \% \mathrm{CO}_{2}$ with DMEM-F12 medium (Gibco; Thermo Fisher Scientific, Inc., Waltham, MA, USA) containing $10 \%$ fetal bovine serum (FBS; Gibco; Thermo Fisher Scientific, Inc.) and $1 \%$ penicillin-streptomycin.

Cell transfection. HK-2 cells were transfected with $100 \mathrm{nM}$ pcDNA3.1, pcDNA-AQP-1, small interfering-negative control RNA (si-NC) or si-AQP-1 for 48 h (Guangzhou RiboBio Co., Ltd., Guangzhou, China) using Lipofectamine ${ }^{\circledR} 3000$ (Invitrogen; Thermo Fisher Scientific, Inc.) according to the manufacturer's protocols. Following transfection, cells were stimulated with $8 \mu \mathrm{g} / \mathrm{ml}$ LPS (Sigma-Aldrich; Merck KGaA, Darmstadt, Germany) and harvested. Total RNA and protein prepared from HK-2 cells were used to check the levels of mRNA or proteins.

$R N A$ extraction and reverse transcription-quantitative polymerase chain reaction ( $R T-q P C R)$. Total RNA was extracted from HK-2 cells using TRIzol reagent (Axygen; Corning Incorporated, Corning, NY, USA) according to the manufacturer's protocols. Purifed RNA $(0.5 \mu \mathrm{g} / \mu \mathrm{l})$ was mixed with nuclease-free water for the cDNA synthesis using a Script cDNA Synthesis kit (Bio-Rad Laboratories, Inc., Hercules, CA, USA) at $37^{\circ} \mathrm{C}$ for $15 \mathrm{~min}$ and $85^{\circ} \mathrm{C}$ for $5 \mathrm{sec}$. RT-qPCR was performed using a Bio-Rad CFX96 real-time PCR System (Bio-Rad Laboratories, Inc.). The qPCR reaction volume was $50 \mu \mathrm{l}$ and included $1 \mu \mathrm{l}$ dNTPs $(10 \mathrm{mM}), 5 \mu \mathrm{l}$ cDNA solution, $1 \mu \mathrm{l}$ of each primer $(50 \mathrm{pM}), 1 \mu \mathrm{l}$ Taq DNA polymerase (Invitrogen; Thermo Fisher Scientific, Inc.), $33 \mu \mathrm{l}$ water, $3 \mu \mathrm{l}$ of $\mathrm{MgCl}_{2}(15 \mathrm{mM})$, and $5 \mu \mathrm{l}$ of PCR buffer (10X). The amplification was performed according to the following condition: Denaturation was performed at $94^{\circ} \mathrm{C}$ for $1 \mathrm{~min}$, annealing at $59^{\circ} \mathrm{C}$ for $1 \mathrm{~min}$ and elongation at $72^{\circ} \mathrm{C}$ for $1 \mathrm{~min}$ for 32 cycles, followed by $72^{\circ} \mathrm{C}$ for $10 \mathrm{~min}$. The expression of AQP-1, AQP-2, AQP-3, AQP-4, AQP-6, AQP-7, AQP-8, AQP-11, TNF- $\alpha$, IL-8, IL- $1 \beta$ and MCP-1 was detected by using the SYBR-Green PCR kit (Takara Bio, Inc., Otsu, Japan). Primers are listed in Table I and GAPDH was used as an internal reference. Results were normalized using the $2^{-\Delta \Delta \mathrm{Cq}}$ method (16) and each experiment was performed in triplicate.

Cell viability. A Cell Counting Kit-8 (CCK-8; Dojindo Molecular Technologies, Inc., Kumamoto, Japan) assay was performed to assess cell viability. Normal or transfected HK-2 cells were seeded in 96-well plates at a density of $2 \times 10^{4}$ cells/well for $24 \mathrm{~h}$. Following LPS treatment, CCK-8 reagents were separately added to well. The cell viability rates were assessed by measuring the optical density at $450 \mathrm{~nm}$ using a microplate reader (Thermo Fisher Scientific, Inc.).

Annexin V-fluorescein isothiocyanate (FITC)/propidium iodide (PI) analysis. Flow cytometry was performed to quantify apoptotic cells using an Annexin V-FITC/PI Apoptosis Detection kit (BioVision, Inc., Milpitas, CA, USA). HK-2 cells were harvested and washed in ice-cold PBS twice and double-stained with Annexin V-FITC and PI at room temperature for $30 \mathrm{~min}$ in the dark. All samples were quantitatively analyzed using a FACSCalibur flow cytometer at $488 \mathrm{~nm}$ emission and $570 \mathrm{~nm}$ excitation (BD Biosciences, San Jose, CA, USA) and analyzed by CellQuest software (version 3.0; BD Biosciences).

Apoptosis detection. According to a previous study (17), apoptosis was determined using a Cell Death Detection ELISA $^{\text {PLus }}$ kit (cat. no. 11774425001; Roche Diagnostics, Basel, Switzerland) that measures cytoplasmic DNA-histone complexes generated during apoptotic DNA fragmentation. Cell apoptosis detection was performed according to the manufacturer's protocol and monitored at $405 \mathrm{~nm}$.

Caspase-3 activity assay. A caspase-3 fluorescent assay kit (Nanjing KeyGen Biotech. Co., Ltd., Nanjing, China) was used to detect caspase activity according to previous study (14). Briefly, cells were lysed using the lysis buffer provided by the kit and centrifuged at $10,000 \mathrm{xg}$ for $1 \mathrm{~min}$ at $4^{\circ} \mathrm{C}$. Supernatants were collected, equal amounts $(30 \mu \mathrm{g})$ of protein were reacted with the synthetic fluorescent substrates, which were provided by the kit, at $37^{\circ} \mathrm{C}$ for $90 \mathrm{~min}$ and fluorescence was measured at $405 \mathrm{~nm}$ using a microplate reader.

Western blot analysis. Following treatment, HK-2 cells were washed twice in cold PBS and lysed in radioimmunoprecipitation assay lysis buffer (EMD Millipore, Billerica, MA, USA) with protease inhibitor cocktail (Roche Diagnostics) to extract proteins. The protein concentration was quantified using a BCA Protein Assay kit (Beyotime Institute of Biotechnology, Haimen, China) and samples (50 $\mu \mathrm{g} /$ lane) were separated by $10 \%$ SDS-PAGE. Blots were transferred to polyvinylidene fluoride membranes (EMD Millipore) bloc which were subsequently blocked in 5\% skimmed milk diluted with TBST at room temperature for $2 \mathrm{~h}$ and incubated overnight at $4^{\circ} \mathrm{C}$ with the following primary antibodies: Anti-AQP-1 antibody (ab15080; 1:1,000; Abcam, Cambridge, MA, USA), phosphorylated (p)-p38 (\#4511), total (t)-p38 (\#8690), p-ERK1/2 (\#8544), t-ERK1/2 (\#4695), p-JNK (\#9251), t-JNK (\#9252), anti-B-cell lymphoma (Bcl)-2 antibody (\#3498) and anti-v-2-associated 
Table I. Primers used for reverse transcription-quantitative polymerase chain reaction.

\begin{tabular}{|c|c|c|}
\hline Gene & Direction & Primer sequence $\left(5^{\prime}-3^{\prime}\right)$ \\
\hline \multirow[t]{2}{*}{ AQP-1 } & Forward & CTGGGCATCGAGATCATCGG \\
\hline & Reverse & ATCCCACAGCCAGTGTAGTCA \\
\hline \multirow[t]{2}{*}{ AQP-2 } & Forward & GCTCCGCTCCATAGCCTTC \\
\hline & Reverse & GGGTGCCAATACCCAAGCC \\
\hline \multirow[t]{2}{*}{ AQP-3 } & Forward & GGGGAGATGCTCCACATCC \\
\hline & Reverse & AAAGGCCAGGTTGATGGTGAG \\
\hline \multirow[t]{2}{*}{ AQP-4 } & Forward & AGCAGTCACAGCGGAATTTCT \\
\hline & Reverse & TCTGTTCCACCCCAGTTGATG \\
\hline \multirow[t]{2}{*}{ AQP-6 } & Forward & GTCTTCGCTTCCACCGACAG \\
\hline & Reverse & GCGGGCTGGATTCATGGAG \\
\hline \multirow[t]{2}{*}{ AQP-7 } & Forward & ACCCGTGGCTCCAAAATGG \\
\hline & Reverse & GGAACCAAGGCCGAATACCA \\
\hline \multirow[t]{2}{*}{ AQP-8 } & Forward & GCGAGTGTCCTGGTACGAAC \\
\hline & Reverse & CAGGCACCCGATGAAGATGAA \\
\hline \multirow[t]{2}{*}{ AQP-11 } & Forward & TGACCCAGTATCACGTCAGC \\
\hline & Reverse & TGACCGCTTTGAGCAAGTCG \\
\hline \multirow[t]{2}{*}{ Tumor necrosis factor- $\alpha$} & Forward & CACCACTTCGAAACCTGGGA \\
\hline & Reverse & TGTAGGCCCCAGTGAGTTCT \\
\hline \multirow[t]{2}{*}{ IL-8 } & Forward & ACCACCGGAAGGAACCATCT \\
\hline & Reverse & AGCACTCCTTGGCAAAACTG \\
\hline \multirow[t]{2}{*}{ IL-1 $\beta$} & Forward & AACCTCTTCGAGGCACAAGG \\
\hline & Reverse & GGCGAGCTCAGGTACTTCTG \\
\hline \multirow[t]{2}{*}{ Monocyte chemoattractant protein-1 } & Forward & GATCTCAGTGCAGAGGCTCG \\
\hline & Reverse & TTTGCTTGTCCAGGTGGTCC \\
\hline \multirow[t]{2}{*}{ GAPDH } & Forward & CАСССАСТССТССАССТТТG \\
\hline & Reverse & CCACCACCCTGTTGCTGTAG \\
\hline
\end{tabular}

$\mathrm{AQP}$, aquaporin; IL, interleukin.

protein X (Bax) antibody (\#2772; all 1:1,000; all Cell Signaling Technology, Inc., Danvers, MA, USA). Membranes were subsequently incubated with a goat anti-mouse IgG conjugated to horseradish peroxidase (\#7076; 1:1,000; Cell Signaling Technology, Inc.) for $2 \mathrm{~h}$ at room temperature. Proteins were visualized using enhanced chemiluminescence-plus reagents (GE Healthcare Life Sciences, Little Chalfont, UK). The density of the bands was measured using the Image $\mathbf{J}$ software (version 1.45s; National Institutes of Health, Bethesda, MA, USA) and values were normalized to the densitometric values of $\alpha$-tubulin (T5168; 1:1,000; Sigma-Aldrich; Merck KGaA), GAPDH (\#5174) or $\beta$-actin (\#3700; both 1:1,000; both Cell Signaling Technology, Inc.).

Measurement of TNF- $\alpha, I L-8, I L-1 \beta$ and MCP-1 levels. The supernatants of HK-2 cells were collected after treatment and the concentrations of TNF- $\alpha$, IL- 8 , IL-1 $\beta$ and MCP-1 were measured using a sandwich ELISA kit (R\&D Systems, Inc., Minneapolis, MN, USA) according to the manufacturer's instruction and as previously described (9). Briefly, the primary antibody (provided in kit) was coated onto ELISA plates and incubated for $2 \mathrm{~h}$ at room temperature. Samples and standards were added to the wells and incubated for $1 \mathrm{~h}$, following which wells were washed and a biotinylated antibody (provided in kit) was added for $1 \mathrm{~h}$ at room temperature. The plates were washed again and streptavidin conjugated to horseradish peroxidase was added for $10 \mathrm{~min}$ at room temperature. Plates were washed and tetramethylbenzidine was added for color development about $30 \mathrm{~min}$ at room temperature and the reaction was terminated with $1 \mathrm{~mol} / \mathrm{l} \mathrm{H}_{2} \mathrm{SO}_{4}$. Absorbance was measured at $490 \mathrm{~nm}$ by using an automated ELISA reader (Thermo Fisher Scientific, Inc.). Concentrations in the samples were calculated using a standard curve and values were expressed as $\mathrm{pg} / \mathrm{ml}$.

Statistical analysis. All statistical analyses were performed using GraphPad Prism 5.0 (GraphPad Software, Inc., La Jolla, CA, USA). Data are presented as the mean \pm standard error of the mean. Data were statistically analyzed using a two-tailed Student's t-test or one-way analysis of variance. $\mathrm{P}<0.05$ was considered to indicate a statistically significant difference.

\section{Results}

LPS-induced injury in HK-2 cells. HK-2 cells were stimulated with $0,1,2,4,8$ and $16 \mu \mathrm{g} / \mathrm{ml}$ LPS for $8 \mathrm{~h}$ and the results 
A

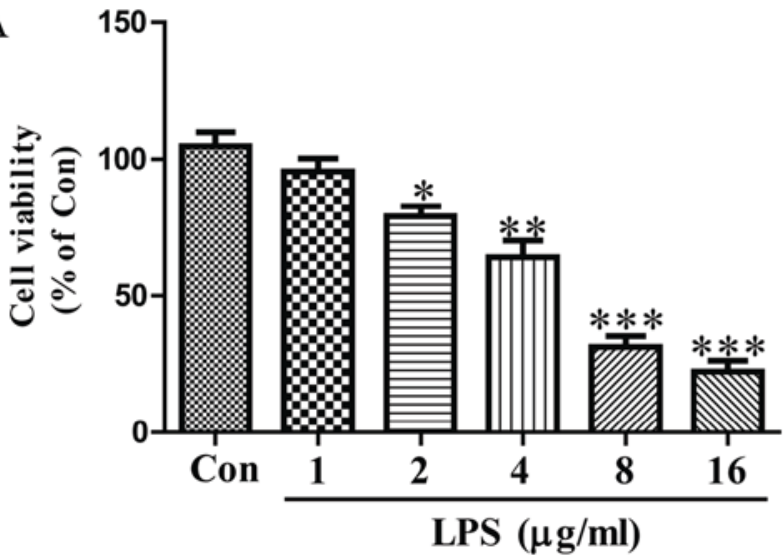

C
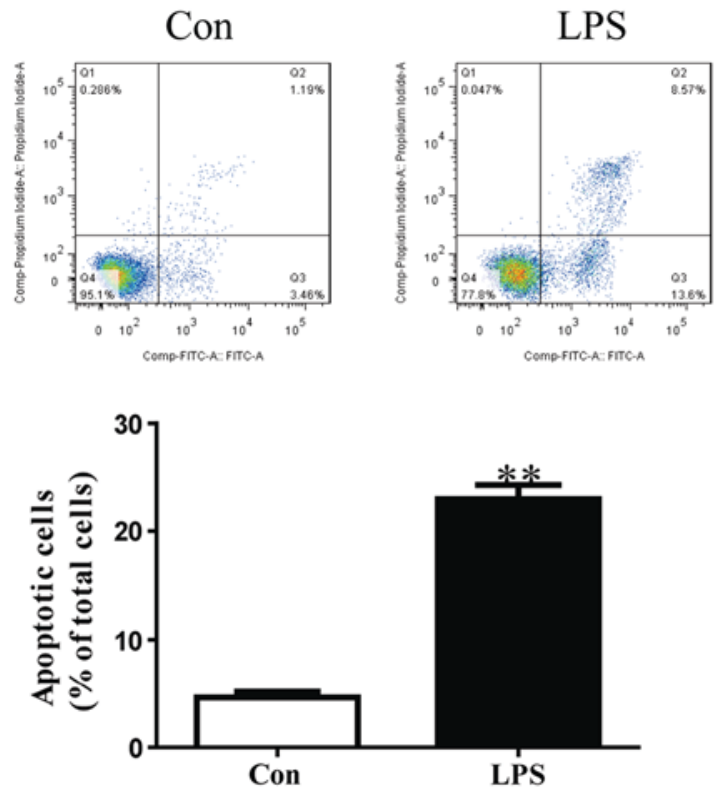

$\mathrm{E}$

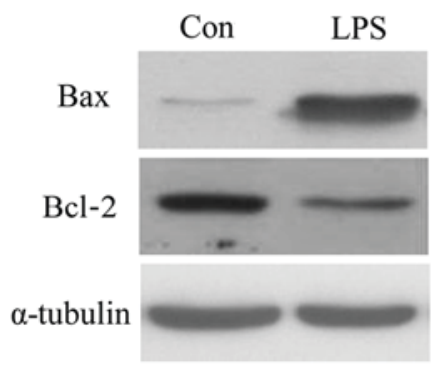

B

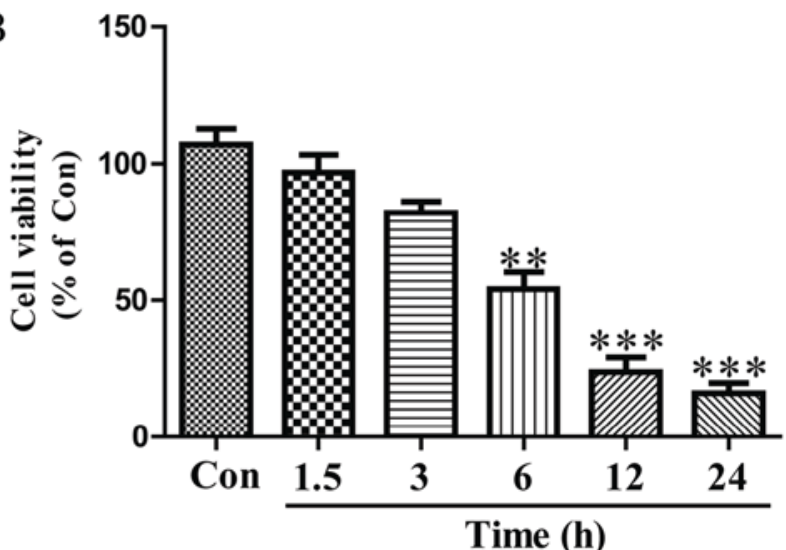

D

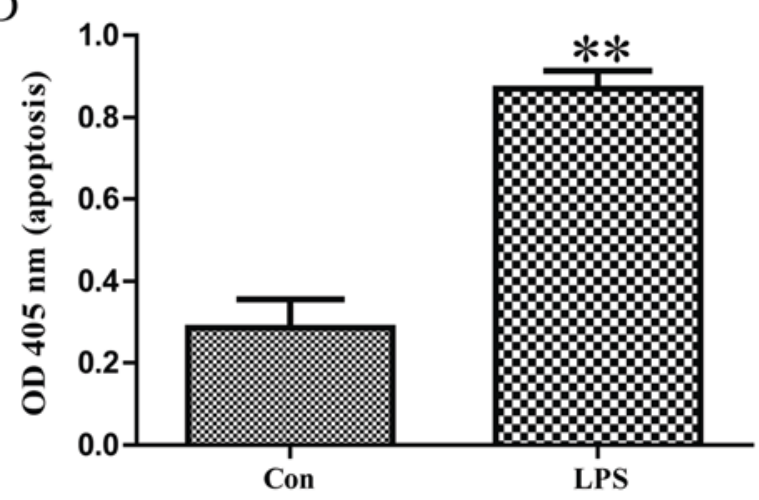

Figure 1. LPS-induced damage in HK-2 cells. HK-2 cells were treated with (A) $0,1,2,4,8$ and $16 \mu \mathrm{g} / \mathrm{ml}$ of LPS for $8 \mathrm{~h}$ or (B) $8 \mu \mathrm{g} / \mathrm{ml} \mathrm{LPS} \mathrm{for} 0,1.5,3,6,12$ and $24 \mathrm{~h}$. HK-2 cells were treated with $8 \mu \mathrm{g} / \mathrm{ml}$ LPS for $12 \mathrm{~h}$ and cell apoptosis was measured by (C) flow cytometric analysis and (D) nucleosomal degradation, respectively. (E) The expression of Bcl-2 and Bax were determined by western blotting. (F) The activity of caspase-3 was determined using a caspase-3 activity detection assay. ${ }^{*} \mathrm{P}<0.05,{ }^{* *} \mathrm{P}<0.01$ and ${ }^{* * * *} \mathrm{P}<0.001$ vs. Con. LPS, lipopolysaccharide; Bcl-2, B-cell lymphoma 2; Bax, Bcl-2-associated protein X; Con, control; FITC, fluorescein isothiocyanate; OD, optical density.

indicated that cell viability was significantly decreased with $2 \mu \mathrm{g} / \mathrm{ml}$ LPS (Fig. 1A). HK-2 cells were stimulated with $8 \mu \mathrm{g} / \mathrm{ml}$ LPS for $0,1.5,3,6,12$ and $24 \mathrm{~h}$ and cell viability was significantly decreased following $6 \mathrm{~h}$ treatment (Fig. 1B). These results suggest that LPS is able to damage HK-2 cells in a concentration- and time-dependent manner and $8 \mu \mathrm{g} / \mathrm{ml}$ LPS treatment for $12 \mathrm{~h}$ was selected for following experiments. Flow cytometry and ELISA assays revealed that LPS dramatically induced apoptosis inHK-2 cells (Fig. 1C and D). Furthermore, Bax expression was increased and Bcl-2 expression was decreased following LPS treatment (Fig. 1E). A caspase-3 assay was performed and the results revealed that LPS treatment significantly increase caspase-3 activity (Fig. 1F). 


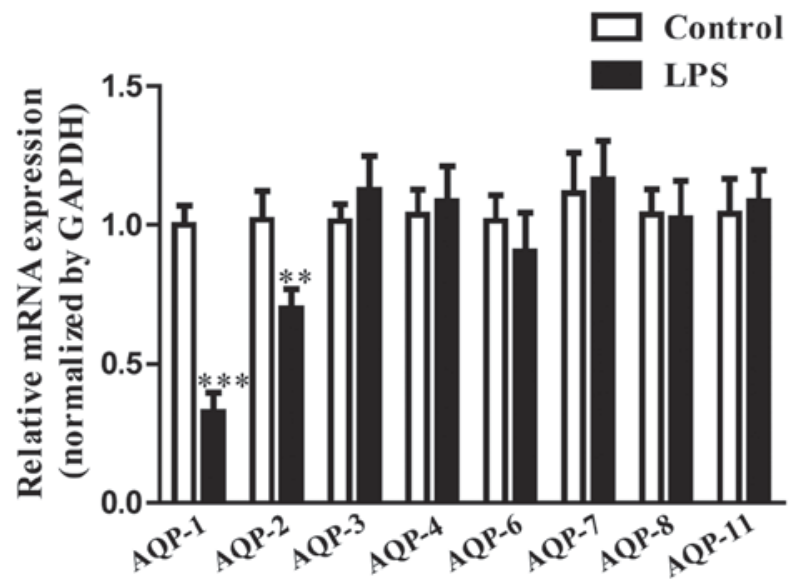

Figure 2. AQP-1 expression was decreased in LPS-induced HK-2 cells. HK-2 cells were treated with $8 \mu \mathrm{g} / \mathrm{ml}$ LPS for $12 \mathrm{~h}$ and the expression of AQP-1, $-2,-3,-4,-6,-7,-8$ and -11 were detected using RT-qPCR. ${ }^{* *} \mathrm{P}<0.01$ and ${ }^{* * *} \mathrm{P}<0.001$ vs. Control. AQP, aquaporin; LPS, lipopolysaccharide.

AQP-1 expression in LPS-induced HK-2 cells. AQP-1, -2, $-3,-4,-6,-7,-8$ and -11 are expressed in the mammalian kidney (13). However, the expression and function of these genes in LPS-stimulated HK-2 cells remain to be elucidated. The results of the present study revealed that AQP-1 mRNA expression levels were the lowest of the AQP genes in LPS-induced HK-2 cells (Fig. 2). AQP-1 was therefore selected for the following experiments.

The effects of AQP-1 in LPS-stimulated apoptosis in HK-2 cells. Following transfection with pcDNA-AQP-1 or si-AQP-1, the expression of AQP-1 was significantly increased or decreased in HK-2 cells, respectively (Fig. 3A). The results revealed that AQP-1 overexpression significantly increased the viability of LPS-induced HK-2 cells (Fig. 3B). Furthermore, AQP-1 overexpression significantly reversed the LPS-induced increase in apoptosis (Fig. 3C). Furthermore, AQP-1 overexpression significantly downregulated Bax expression and upregulated $\mathrm{Bcl} 2$ expression (Fig. 3D), as well as inhibiting caspase-3 activity in LPS-induced HK-2 cells (Fig. 3E). However, AQP-1 knockdown aggravated the pro-apoptotic effect of LPS on HK-2 cells (Fig. 3A-E). These results indicate that AQP-1 overexpression is able to effectively protect HK-2 cells from LPS-induced apoptosis.

Effect of AQP-1 on LPS-induced inflammatory cytokine and chemokine expression in $H K-2$ cells. The expression levels of TNF- $\alpha$, IL-8, IL-1 $\beta$ and MCP-1 mRNA and protein were significantly upregulated in LPS-induced HK-2 cells (Figs. 4 and 5). LPS-induced increases in TNF- $\alpha$, IL-8, IL-1 $\beta$ and MCP-1 mRNA and protein were significantly aggravated in si-AQP-1-transfected HK-2 cells (Fig. 4). However, the effects of LPS on TNF- $\alpha$, IL-8, IL-1 $\beta$ and MCP-1 were significantly reduced in pcDNA-AQP-1-transfected HK-2 cells (Fig. 5).

Effects of AQP-1 on LPS-induced renal MAPK p38, ERK1/2 and JNK signaling pathways. The effects of AQP-1 on LPS-stimulated MAPK p38, ERK1/2 and JNK phosphorylation in HK-2 cells were assessed. The results revealed that
AQP-1 knockdown increased the LPS-induced upregulation of p-p38 and p-ERK1/2 (Fig. 6A). By contrast, the expression of p-p38 and p-ERK1/2 was significantly reduced in HK-2 cells followed by transfection with pcDNA-AQP-1 (Fig. 6B). The expression of t-p38 and t-ERK1/2 protein was not affected. No significant differences in $\mathrm{p}-\mathrm{JNK}$ or t-JNK were observed between groups (Fig. 6A and B). These results suggest that AQP-1 may protect HK-2 cells against LPS-induced inflammation and apoptosis via inhibiting the p38 and ERK1/2 signaling pathways.

\section{Discussion}

The aim of the present study was to determine the functional roles of AQP-1 in LPS-stimulated HK-2 cells, an in vitro septic acute kidney injury model of DIC, and to elucidate the mechanism of AQP-1 in this model. The results revealed that LPS treatment decreased HK-2 cell viability as well as increasing apoptosis and the expression of proinflammatory cytokines and chemokines. AQP-1 upregulation served a protective role by reversing LPS-induced damage in HK-2 cells. Furthermore, AQP-1 was able to modulate the p38 and ERK1/2 signaling pathways.

It has been reported that LPS treatment induces apoptosis and caspase-3 cleavage in tubular epithelial cells (18). In accordance with previous reports, the results of the present study demonstrated that LPS treatment dramatically reduced the viability of HK-2 cells and induced cell apoptosis (8). Previous studies have reported that the expression of AQP-1 and AQP-5 was significantly reduced in rats with LPS-induced acute lung injury (19-22). Furthermore, Jin et al (23) demonstrated that the expression of AQP5 was downregulated in a rat model of LPS-induced lung DIC (23). The expression of AQP-2 has been reported to be decreased in rats with LPS-induced acute kidney injury (24). However, the expression and role of AQPs in LPS-induced acute kidney injury remain to be elucidated. In the present study, the expression levels of AQPs in LPS-stimulated HK-2 cells were assessed in vitro and the results revealed that AQP-1 levels were the lowest of the eight AQPs expressed in HK-2 cells. The effects of LPS on cell viability and apoptosis were partially reversed by AQP-1 overexpression, suggesting that AQP-1 protects against LPS-induced injury in HK-2 cells. It was also demonstrated AQP-1 knockdown aggravated the LPS-induced decrease in the $\mathrm{Bax} / \mathrm{Bcl}-2$ ratio and activated caspase-3, whereas overexpression of AQP-1 inhibited LPS-induced apoptosis by increasing the Bax/Bcl-2 ratio and increasing caspase 3 activity.

It has been reported that LPS stimulation increases the expression of proinflammatory cytokines and chemokines, including TNF- $\alpha$, IL-1 $\beta$ and IL- 6 in human HK- 2 cells, resulting in endotoxemia and sepsis (25). The results of the present study support these findings. AQP-1 overexpression partially the effects of LPS, whereas AQP-1 knockdown aggravated them. The results of the present study suggest that AQP-1 may serve an anti-inflammatory role in LPS-stimulated septic acute kidney injury.

$\mathrm{NF}-\kappa \mathrm{B}$ is a well-known transcription factor that regulates the expression of multiple inflammation-associated proteins in response to various infections (26). ERK, p38 and c-JNK are members of the MAPK family and are able to activate 
A

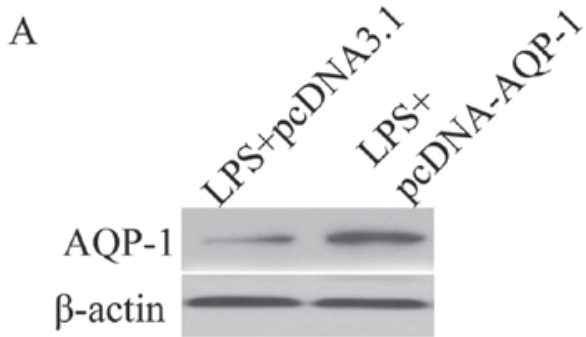

$\mathrm{B}$

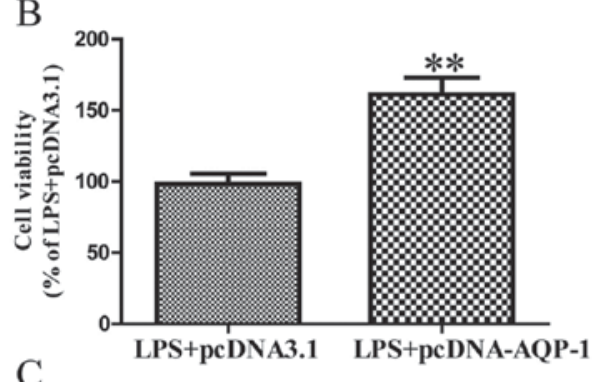

C

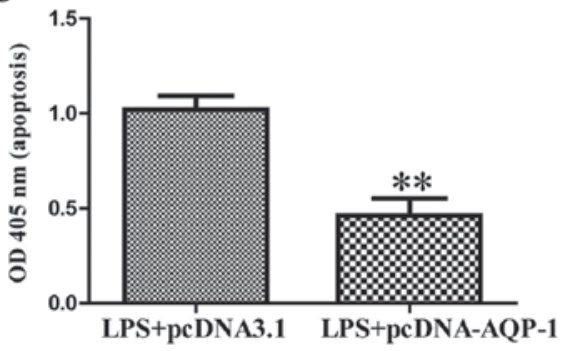

$\mathrm{D}$

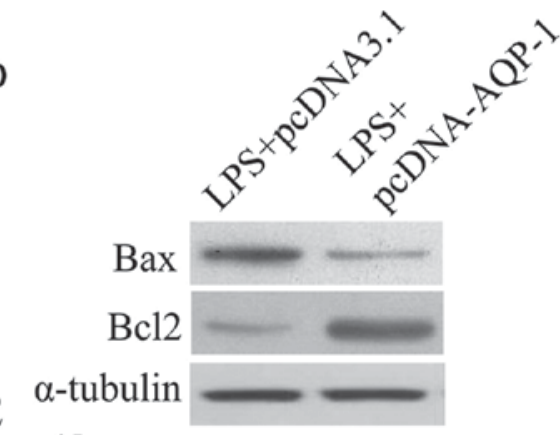

$\mathrm{E}$

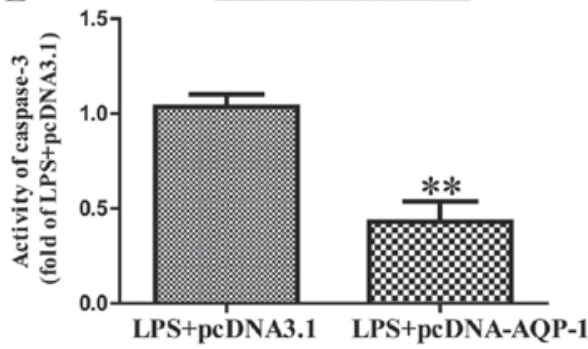

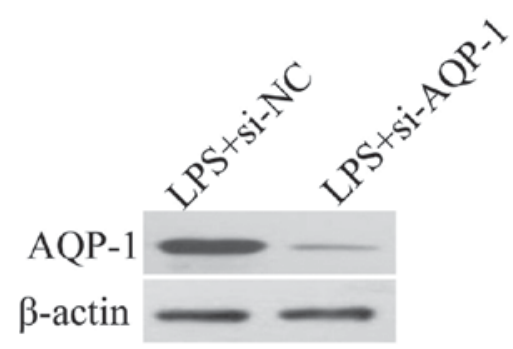
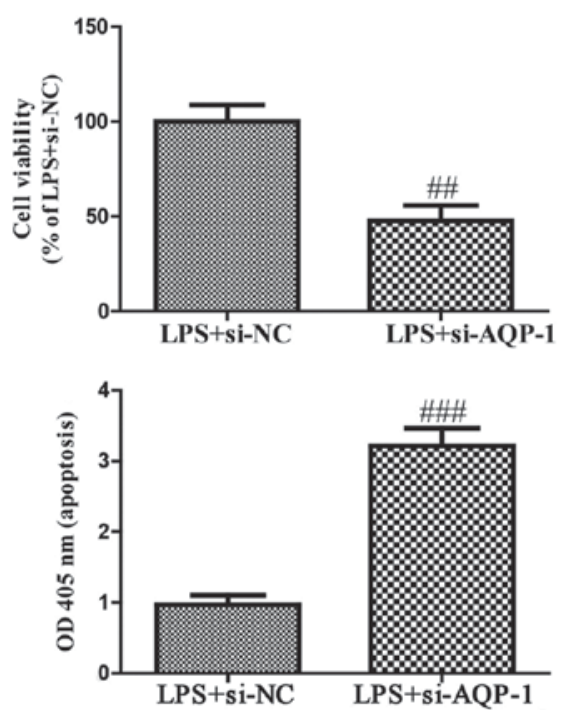

Bax

$\mathrm{Bcl}-2$

$\alpha$-tubulin

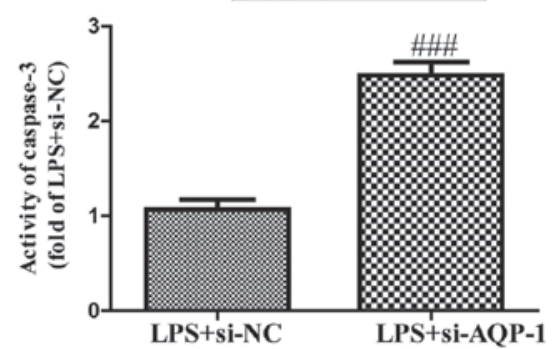

Figure 3. Effects of AQP-1 expression on LPS-induced apoptosis in HK-2 cells. Cells were transfected with pcDNA-AQP-1 or si-AQP-1 and treated with $8 \mu \mathrm{g} / \mathrm{ml}$ LPS for $12 \mathrm{~h}$. (A) The expression of AQP-1 was determined using western blotting. (B) Cell viability was assessed using a Cell Counting Kit-8 assay. (C) Cell apoptosis was measured using an ELISA kit. (D) The expression of Bcl-2 and Bax was determined using western blotting. (E) The activity of caspase-3 was determined using a caspase-3 activity detection assay. ${ }^{\# \#} \mathrm{P}<0.01$ and ${ }^{\# \# \#} \mathrm{P}<0.001$ vs. si-NC; ${ }^{* *} \mathrm{P}<0.01$ vs. pcDNA3.1. AQP, aquaporin; LPS, lipopolysaccharide; si, small interfering RNA; Con, control; NC, negative control; Bcl-2, B-cell lymphoma 2; Bax, Bcl-2-associated X protein; OD, optical density.

NF- $\kappa B$ (27). ERK1/2 activation may further activate and translocate NF- $\kappa B$ to the nucleus (28). Furthermore, LPS-induced NF- $\kappa \mathrm{B}$ activation may be associated with the phosphorylation of MAPKs. In the present study it was demonstrated that AQP-1 overexpression was able to significantly reduce the phosphorylation of p38 and ERK1/2, whereas AQP-1 enhanced the phosphorylation of $\mathrm{p} 38$ and ERK1/2. However, the phosphorylation of JNK was not affected by AQP-1. These results suggest that AQP-1 exerts a cytoprotective role in LPS-induced HK-2 cells via the p38 and ERK1/2 signaling pathways.

In conclusion, the results of the present study suggest that AQP-1 overexpression is able to partially reverse the LPS-induced decrease in cell viability and increase in apoptosis, as well as reducing inflammation in HK-2 cells. AQP-1 may have anti-apoptotic and anti-inflammatory functional 

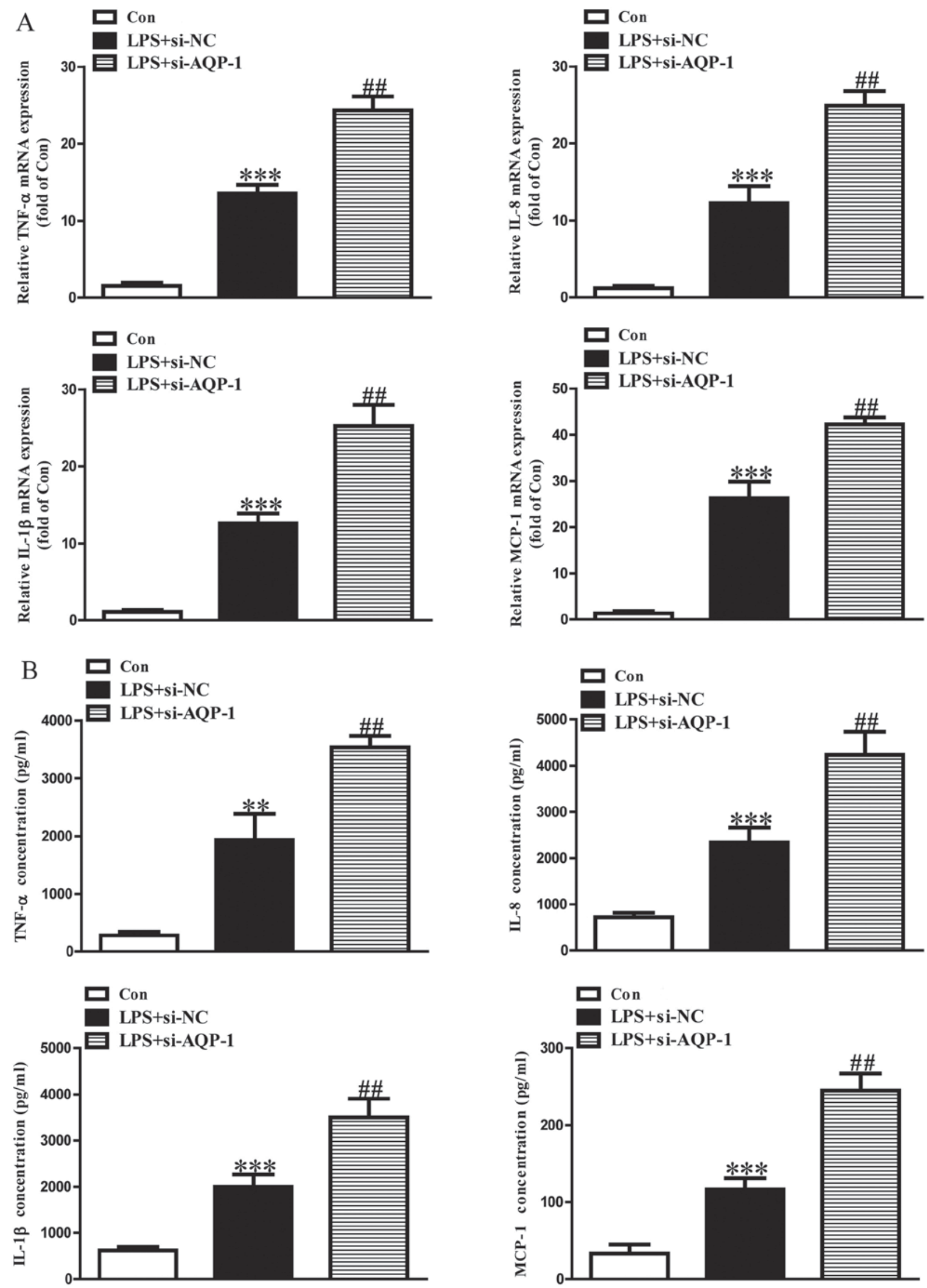

Figure 4. Effects of AQP-1 knockdown on LPS-induced inflammatory cytokine and chemokine expression in HK-2 cells. Following transfection with si-AQP-1, HK-2 cells were treated with $8 \mu \mathrm{g} / \mathrm{ml}$ LPS for $12 \mathrm{~h}$. The expression levels of TNF- $\alpha$, IL- 8 , IL-1 $\beta$ and MCP-1 protein and mRNA were determined by (A) reverse transcription-quantitative polymerase chain reaction and (B) ELISA, respectively. The Con group was treated with vehicle alone. ${ }^{* *} \mathrm{P}<0.01$ and ${ }^{* * * *} \mathrm{P}<0.001$ vs. Con. ${ }^{\# \#} \mathrm{P}<0.01$ vs. LPS+si-NC. AQP, aquaporin; LPS, lipopolysaccharide; si, small interfering RNA; TNF, tumor necrosis factor; IL, interleukin; MCP, monocyte chemoattractant protein; Con, control; NC, negative control. 

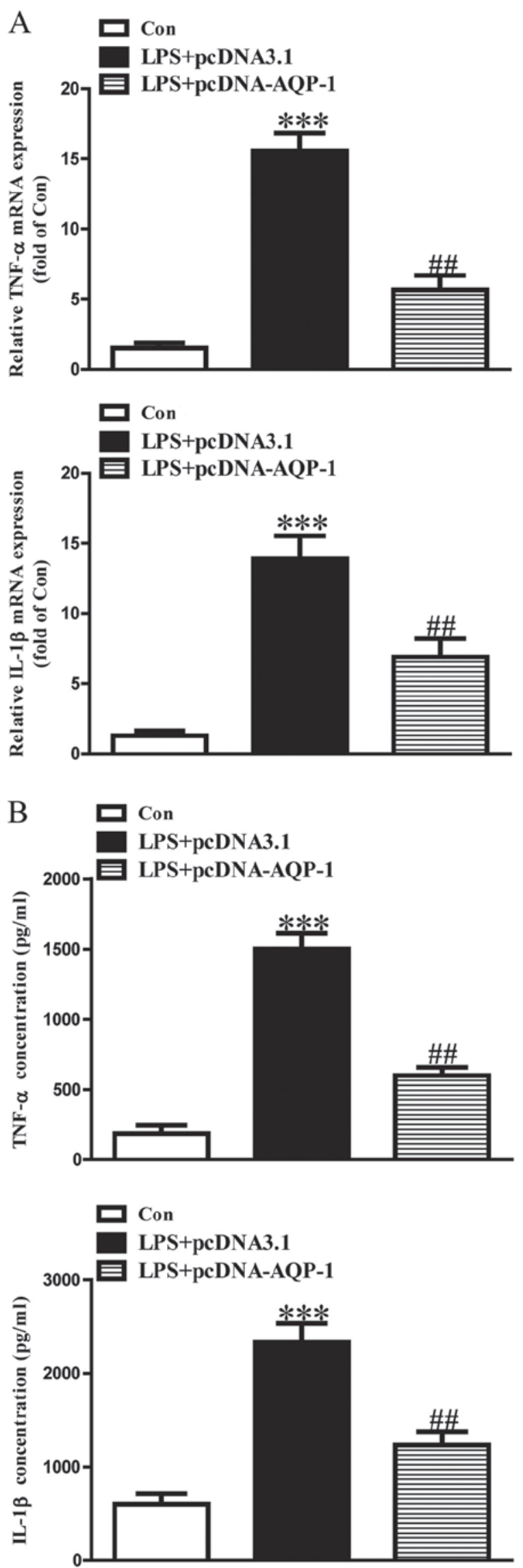
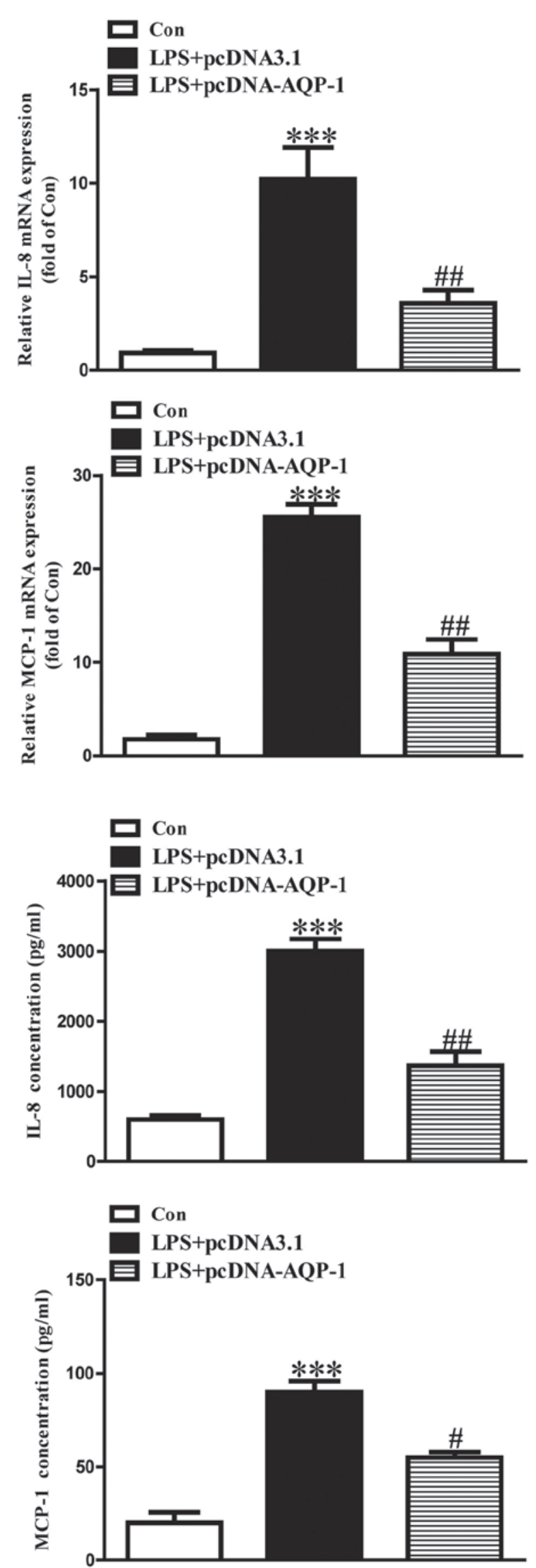

Figure 5. Effects of AQP-1 overexpression on LPS-induced inflammatory cytokines and chemokines in HK-2 cells. Following transfection with pcDNA-AQP-1, HK-2 cells were treated with $8 \mu \mathrm{g} / \mathrm{ml}$ LPS for $12 \mathrm{~h}$. The expression levels of TNF- $\alpha$, IL- 8 , IL-1 $\beta$ and MCP-1 protein and mRNA were determined by (A) reverse transcription-quantitative polymerase chain reaction and (B) ELISA, respectively. The Con group was treated with vehicle alone. ${ }^{* * * *} \mathrm{P}<0.001$ vs. Con. ${ }^{~} \mathrm{P}<0.05$ and ${ }^{\# /} \mathrm{P}<0.01$ vs. LPS+pcDNA3.1. AQP, aquaporin; LPS, lipopolysaccharide; TNF, tumor necrosis factor; IL, interleukin; MCP, monocyte chemoattractant protein; Con, control; NC, negative control; si, small interfering RNA. 
A
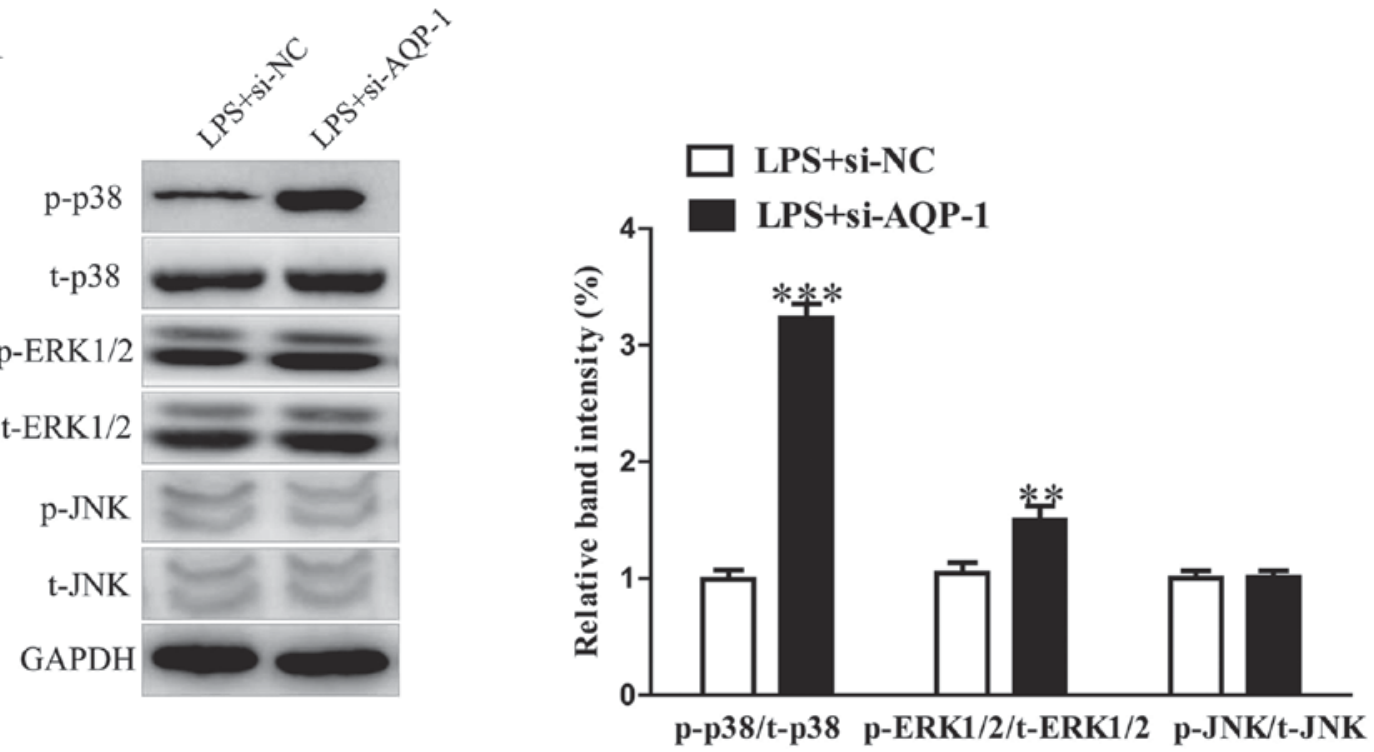

B
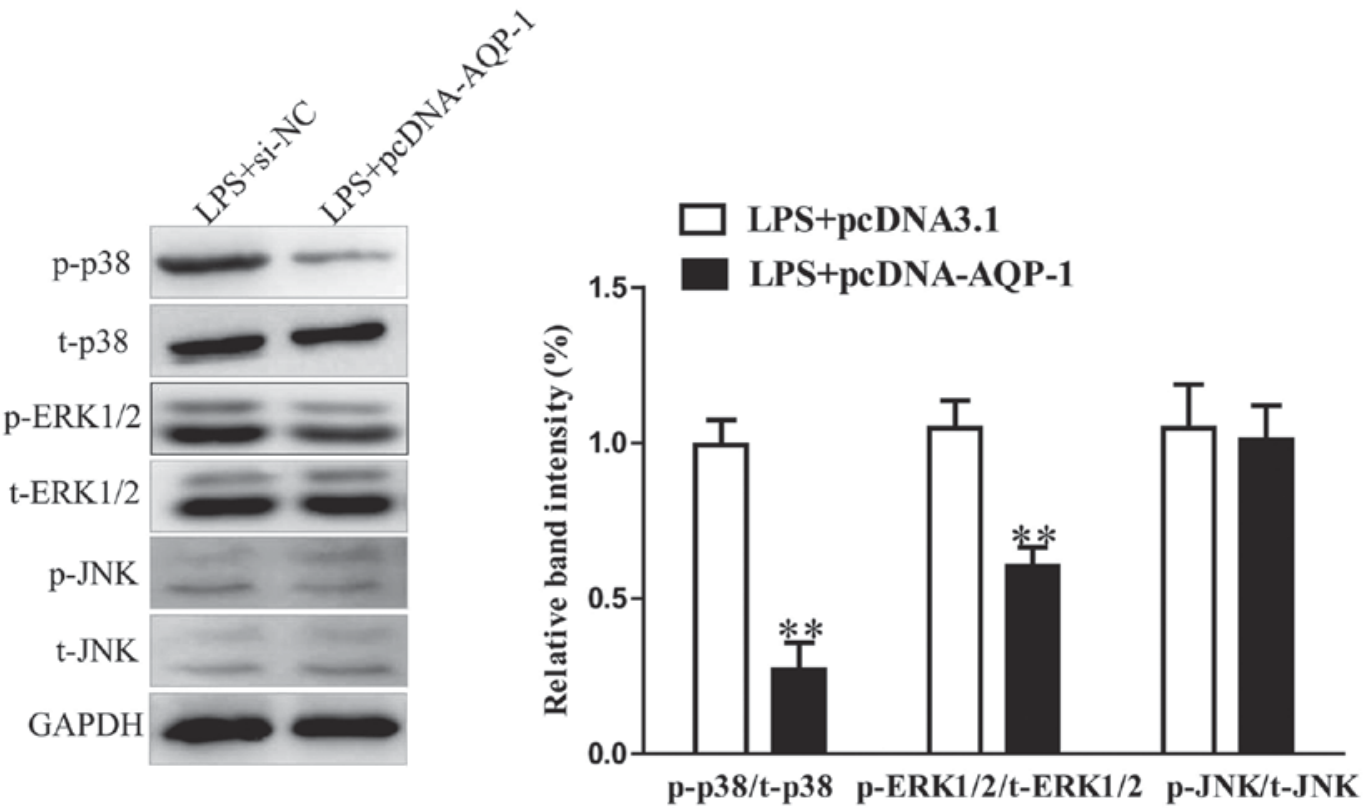

Figure 6. Effects of AQP-1 on LPS-activated renal MAPK p38, ERK1/2 and JNK signaling. Following transfection with pcDNA-AQP-1 or si-AQP-1, HK-2 cells were treated with $8 \mu \mathrm{g} / \mathrm{ml}$ LPS for $12 \mathrm{~h}$. (A and B) The p-p38, t-p38, p-ERK1/2, t-ERK1/2, p-JNK and t-JNK protein levels were measured using western blotting. ${ }^{* *} \mathrm{P}<0.01,{ }^{* * * *} \mathrm{P}<0.001$ vs. LPS+pcDNA3.1 or LPS+si-NC. AQP, aquaporin; LPS, lipopolysaccharide; MAPK, mitogen activated protein kinase; ERK, extracellular signal-regulated kinase; JNK, c-JUN N-terminal kinase; si, small interfering RNA; p, phosphorylated; t, total; NC, negative control.

roles in HK-2 cells and these effects may be achieved via the p38 and ERK1/2 signaling pathways. These results may provide a basis for the used of AQP-1 as a treatment for septic acute kidney injury in DIC.

\section{Acknowledgements}

Not applicable.

\section{Funding}

This study was supported by the Natural Youth Science Foundation of China (grant no. 81501825) and Youth Science Foundation of Heilongjiang Province of China Grant (grant no. QC2012C035).

\section{Availability of data and materials}

The datasets used and/or analyzed during the current study are available from the author for correspondence upon reasonable request.

\section{Authors' contributions}

YW and WZ performed the experiments. GY and QL analyzed the data. YJ made substantial contributions to conception and design, acquisition of data, analysis and interpretation of data, acquisition of funding. YW and YJ was involved in drafting the manuscript and revising it critically for important intellectual content. All authors gave their final approval of the version to be published. YJ agreed to be accountable for all 
aspects of the work in ensuring that questions related to the accuracy or integrity of any part of the work are appropriately investigated and resolved.

\section{Ethics approval and consent to participate}

Not applicable.

\section{Consent for publication}

Not applicable.

\section{Competing interests}

The authors declare that they have no competing interests.

\section{References}

1. Minomo H, Inoue K, Sakaki S, Okazaki T, Kobayashi K, Inoue $\mathrm{K}$ and Miyata A: Establishment of disseminated intravascular coagulation (DIC) model by a single iv administration of Escherichia coli-derived lipopolysaccharide (LPS) to cynomolgus monkeys and evaluation of its pathophysiological status. J Pharmacol Sci 133: 88-95, 2017.

2. Asakura H: Classifying types of disseminated intravascular coagulation: Clinical and animal models. J Intensive Care 2: 20, 2014

3. Fink MP: Animal models of sepsis. Virulence 5: 143-153, 2014.

4. Wigton DH, Kociba GJ and Hoover EA: Infectious canine hepatitis: Animal model for viral-induced disseminated intravascular coagulation. Blood 47: 287-296, 1976.

5. Song J, Hu D, He C, Wang T, Liu X, Ma L, Lin Z and Chen Z: Novel biomarkers for early prediction of sepsis-induced disseminated intravascular coagulation in a mouse cecal ligation and puncture model. J Inflamm (Lond) 10: 7, 2013.

6. Singer M, Deutschman CS, Seymour CW, Shankar-Hari M, Annane D, Bauer M, Bellomo R, Bernard GR, Chiche JD, Coopersmith CM, et al: The third international consensus definitions for sepsis and septic shock (Sepsis-3). JAMA 315: 801-810, 2016 .

7. Angus DC and van der Poll T: Severe sepsis and septic shock. N Engl J Med 369: 840-851, 2013.

8. Li C, Wu J, Li Y and Xing G: Cytoprotective effect of heat shock protein 27 against lipopolysaccharide-induced apoptosis of renal epithelial HK-2 cells. Cell Physiol Biochem 41: 2211-2220, 2017.

9. Zhong F, Chen H, Han L, Jin Y and Wang W: Curcumin attenuates lipopolysaccharide-induced renal inflammation. Biol Pharm Bull 34: 226-232, 2011.

10. Bascands JL, Bachvarova M, Neau E, Schanstra JP and Bachvarov D: Molecular determinants of LPS-induced acute renal inflammation: Implication of the kinin $\mathrm{B} 1$ receptor. Biochem Biophys Res Commun 386: 407-412, 2009.

11. Zhang WJ, Wei H, Hagen $\mathrm{T}$ and Frei B: Alpha-lipoic acid attenuates LPS-induced inflammatory responses by activating the phosphoinositide 3-kinase/Akt signaling pathway. Proc Natl Acad Sci USA 104: 4077-4082, 2007.

12. Lee S, Kim W, Kang KP, Moon SO, Sung MJ, Kim DH, Kim HJ and Park SK: Agonist of peroxisome proliferator-activated receptor-gamma, rosiglitazone, reduces renal injury and dysfunction in a murine sepsis model. Nephrol Dial Transplant 20 1057-1065, 2005.

13. Nielsen S, Kwon TH, Frøkiaer J and Agre P: Regulation and dysregulation of aquaporins in water balance disorders. J Intern Med 261: 53-64, 2007.
14. Marrone J, Danielli M, Gaspari CI and Marinelli RA: Adenovirus-mediated human aquaporin-1 expression in hepatocytes improves lipopolysaccharide-induced cholestasis. IUBMB Life 69: 978-984, 2017.

15. Li J, Zhang M, Mao Y, Li Y, Zhang X, Peng X and Yu F: The potential role of aquaporin 1 on aristolochic acid I induced epithelial mesenchymal transition on HK-2 cells. J Cell Physiol 233: 4919-4925, 2018

16. Livak KJ and Schmittgen TD: Analysis of relative gene expression data using real-time quantitative PCR and the 2(-Delta Delta C(T)) method. Methods 25: 402-408, 2001.

17. Zhao Z, Li C, Xi H, Gao Y and Xu D: Curcumin induces apoptosis in pancreatic cancer cells through induction of forkhead box ol (FOXO1) and inhibition of PI3K/Akt pathway. Mol Med Rep 12 5415-5422, 2015.

18. Li S, Guo L, Qian P, Zhao Y, Liu A, Ji F, Chen L, Wu X and Qian G: Lipopolysaccharide induces autophagic cell death through the PERK-dependent branch of the unfolded protein response in human alveolar epithelial A549 cells. Cell Physiol Biochem 36: 2403-2417, 2015.

19. Jiao G, Li E and Yu R: Decreased expression of AQP1 and AQP5 in acute injured lungs in rats. Chin Med J (Engl) 115: 963-967, 2002.

20. Su X, Song Y, Jiang J and Bai C: The role of aquaporin-1 (AQP1) expression in a murine model of lipopolysaccharideinduced acute lung injury. Respir Physiol Neurobiol 142: 1-11, 2004.

21. Jiang YX, Dai ZL, Zhang XP, Zhao W, Huang Q and Gao LK: Dexmedetomidine alleviates pulmonary edema by upregulating AQP1 and AQP5 expression in rats with acute lung injury induced by lipopolysaccharide. J Huazhong Univ Sci Technolog Med Sci 35: 684-688, 2015.

22. Hong-Min F, Chun-Rong H, Rui Z, Li-Na S, Ya-Jun W and Li L CGRP 8-37 enhances lipopolysaccharide-induced acute lung injury and regulating aquaporin 1 and 5 expressions in rats. J Physiol Biochem 73: 381-386, 2016.

23. Jin Y, Yu G, Peng P, Zhang Y and Xin X: Down-regulated expression of AQP5 on lung in rat DIC model induced by LPS and its effect on the development of pulmonary edema. Pulm Pharmacol Ther 26: 661-665, 2013.

24. Cui WY, Tian AY and Bai T: Protective effects of propofol on endotoxemia-induced acute kidney injury in rats. Clin Exp Pharmacol Physiol 38: 747-754, 2011.

25. Hirschfeld M, Ma Y, Weis JH, Vogel SN and Weis JJ: Cutting edge: Repurification of lipopolysaccharide eliminates signaling through both human and murine toll-like receptor 2 . J Immunol 165: 618-622, 2000.

26. Lerolle N, Nochy D, Guérot E, Bruneval P, Fagon JY, Diehl JL and Hill G: Histopathology of septic shock induced acute kidney injury: Apoptosis and leukocytic infiltration. Intensive Care Med 36: 471-478, 2010.

27. Li Y, Chen L, Wang C, Chen J, Zhang X, Hu Y, Niu X, Pei D, $\mathrm{He} \mathrm{Z}$ and $\mathrm{Bi} \mathrm{Y}$ : Extracellular matrix metalloproteinase inducer enhances host resistance against pseudomonas aeruginosa infection through MAPK signaling pathway. Am J Transl Res 8: 5619-5627, 2016

28. Soubh AA, Abdallah DM and El-Abhar HS: Geraniol ameliorates TNBS-induced colitis: Involvement of $\mathrm{Wnt} / \beta$-catenin, p38MAPK, NFкB, and PPAR $\gamma$ signaling pathways. Life Sci 136: $142-150,2015$.

This work is licensed under a Creative Commons Attribution-NonCommercial-NoDerivatives 4.0 International (CC BY-NC-ND 4.0) License. 